\title{
Use of Goal Setting to Enhance Self-Efficacy After Sports-Related Injury: A Critically Appraised Topic
}

\author{
Caitlin Brinkman, Shelby E. Baez, Francesca Genoese, and Johanna M. Hoch
}

\begin{abstract}
Clinical Scenario: Patients after sports-related injury experience deficits in self-efficacy. Goal setting may be an appropriate psychoeducation technique to enhance self-efficacy after sports-related injury. Clinical Question: Does goal setting-enhanced rehabilitation improve self-efficacy compared with traditional rehabilitation alone in individuals with sports-related injury? Summary of Key Findings: Two randomized controlled trials were included. The two studies selected assessed changes in self-efficacy before and after a goal-setting intervention following sports-related injury in an athletic population. Both studies used the Sports Injury Rehabilitation Beliefs Survey to evaluate self-efficacy. Clinical Bottom Line: There is currently consistent, goodquality, patient-oriented evidence that supports the use of goal setting to improve self-efficacy in patients undergoing rehabilitation for sports-related injury compared with the standard of care group. Future research should examine optimal timing for the implementation of goal setting in order to enhance self-efficacy following sports-related injury. Strength of Recommendation: The grade of A is recommended by the Strength of Recommendation Taxonomy for consistent, good-quality, patient-oriented evidence.
\end{abstract}

Keywords: rehabilitation, goal setting, interprofessional collaboration

\section{Clinical Scenario}

Nearly 4 million sports-related injuries occur each year. ${ }^{1}$ Sportsrelated injuries often require musculoskeletal rehabilitation to allow athletes to return to previous levels of sports participation. ${ }^{2}$ Throughout the rehabilitation process, emphasis is traditionally placed on addressing physical impairments and limitations, such as deficits in range of motion and strength. ${ }^{2}$ However, the impact of psychological factors on the rehabilitation process and health outcomes after sports-related injury has been increasingly explored. Self-efficacy is an individual's belief in their ability to carry out a task; this belief is different from whether one can perform the task. ${ }^{3}$ This psychological factor can affect health outcomes after sports-related injury. ${ }^{3}$ Increased levels of selfefficacy have been found to be associated with increased adherence to rehabilitation, ${ }^{2,4}$ and self-efficacy may also be a stronger determinant of disability than other clinical outcome measures. ${ }^{5}$ Therefore, it is important to include interventions to improve selfefficacy during the rehabilitation process, in order to positively influence health outcomes following sports-related injury. ${ }^{5}$ Multiple cognitive behavioral interventions have been developed to increase self-efficacy, ${ }^{6}$ but an intervention that is frequently utilized in rehabilitation after sports-related injury is goal setting. ${ }^{7}$ However, only a limited amount of literature has explored the use of goal setting as an intervention for enhancing self-efficacy in sports-related injury rehabilitation programs. Therefore, the purpose of this critically appraised topic is to critically appraise and synthesize the available evidence examining the effectiveness of goal setting-enhanced rehabilitation in improving self-efficacy in individuals with sports-related injury.

Brinkman and Genoese are with the Post-Professional Athletic Training Program, University of Kentucky, Lexington, KY. Baez is with the Department of Kinesiology, Michigan State University, East Lansing, Michigan. Hoch is with the Department of Athletic Training and Clinical Nutrition, University of Kentucky, Lexington, KY. Brinkman (c.brinkman@uky.edu) is corresponding author.

\section{Focused Clinical Question}

Does goal setting-enhanced rehabilitation improve self-efficacy compared with traditional rehabilitation alone in individuals with sports-related injury?

\section{Search Strategy}

A computerized search was completed in May 2019. The search terms used were:

- Patient/client group: individuals with sports-related injury

- Intervention: goal setting

- Comparison: traditional rehabilitation

- Outcomes: self-efficacy

\section{Sources of Evidence Searched}

- EBSCOhost

O CINAHL with full text

O MEDLINE

O Psychology \& Behavioral Sciences Collection

O PsychINFO

o SPORTDiscus

\section{Inclusion and Exclusion Criteria}

The inclusion criteria for study were as follows:

- studies that were classified as level 2 evidence or higher;

- studies that included participants from athletic populations (ie, traditional athletics, tactical athletes) between 14 and 60 years of age;

- studies that included participants who sustained sports-related injury; 
- studies that assessed self-efficacy using a patient-reported outcome measure (eg, Sports Injury Rehabilitation Beliefs Survey [SIRBS]); and

- studies published in English.

The exclusion criteria for the study were as follows:

- studies that examined the effectiveness of other psychological interventions (eg, modeling, imagery) on self-efficacy;

- studies that examined the effectiveness of goal setting in nonathletic populations (eg, disability); and

- studies that examined the effectiveness of goal setting in injuries unrelated to sports participation (eg, motor vehicle accident).

\section{Evidence Quality Assessment}

- The Physiotherapy Evidence Database scale was used to determine the quality of the randomized clinical trials selected for this critically appraised topic (CAT). Two authors (C.B. and F.G.) independently reviewed and scored each study. After review, the authors met to review each study and come to a consensus on the quality.

\section{Summary of Search, "Best" Evidence Appraised, and Key Findings}

- The literature search yielded 17 studies (Figure 1). After review of the abstracts, 15 studies were excluded for not meeting the inclusion criteria for this CAT. Two randomized controlled trials ${ }^{7,8}$ met the inclusion criteria for this CAT and were critically appraised. Table 1 contains study characteristics for both studies included.

- The 2 studies selected assessed changes in self-efficacy before and after a goal-setting intervention following sports-related injury in an athletic population. One study examined the effectiveness of goal setting in tactical athletes with low back pain. ${ }^{8}$ The second study examined the relationship in patients with a variety of musculoskeletal injuries that required operative procedure. ${ }^{7}$

- Both of the studies used the SIRBS ${ }^{9}$ - specifically, they used the 4-item self-efficacy subscale-to evaluate self-efficacy after successfully completing all rehabilitation exercises.

- The results of Evans and Hardy ${ }^{7}$ demonstrated a significant effect for group $(P=.002)$; however, the post hoc analysis did not

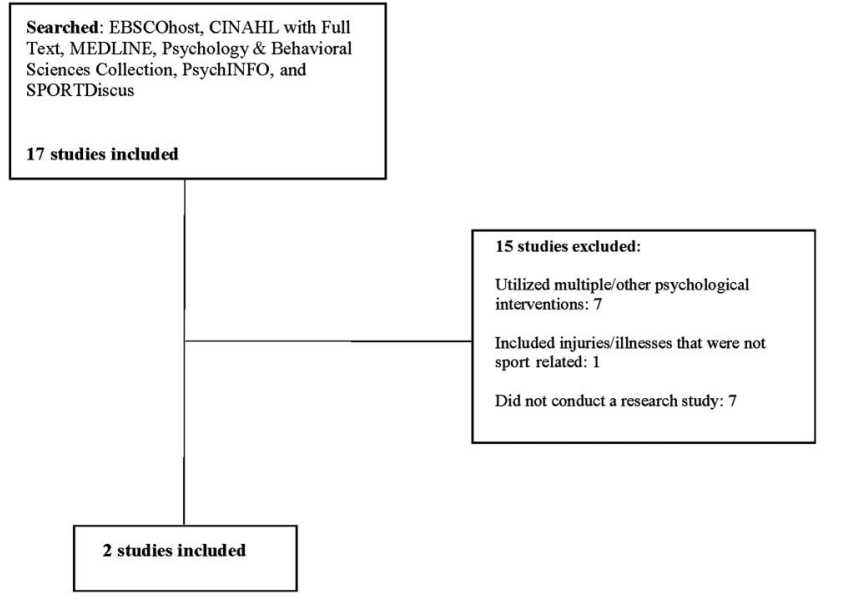

Figure 1 - Summary of search history and included studies. identify any significant differences between the 3 groups (goalsetting intervention group, social support group, or control group). However, we calculated Hedge $g$ effect sizes ${ }^{10}$ to determine the magnitude of the difference between posttreatment self-efficacy scores between the groups. Our results indicated a strong effect (effect size $=0.76 ; 95 \%$ confidence interval, -0.04 to 1.55 ) between the goal-setting group $($ week 8 postmean $=25.54$ [2.73] $)$ and the social-support control group (week 8 postmean $=22.69$ [4.39]), and a strong effect (effect size $=2.15$; 95\% confidence interval, 1.18 to 3.11 ) between the goal-setting group and the control group (week 8 postmean $=18.54$ [3.53]).

- Furthermore, we calculated Hedge $g$ effect sizes to determine the magnitude of the difference between posttreatment and pretreatment self-efficacy scores within each group included in Evans and Hardy ${ }^{7}$ Our results indicated a moderate positive effect size for the goal-setting group (effect size $=0.61 ; 95 \%$ confidence interval, -0.18 to 1.40 ), a weak negative effect size for the social support group (effect size $=-0.38$; 95\% confidence interval, -1.16 to 0.40 ), and a strong negative effect size for the control group (effect size $=-0.77 ; 95 \%$ confidence interval, -1.57 to 0.03 ). Caution must be exercised in interpreting these effect sizes, because the confidence interval for each effect size did cross 0.

- Coppack et $\mathrm{al}^{8}$ identified a significant group $\times$ time effect $(P<.001)$. They did not present specific post hoc results between the groups. However, our calculated effect sizes revealed a strong effect (effect size $=0.93 ; 95 \%$ confidence interval, 0.23 to 1.69$)$ between the experimental goal-setting group (postmean $=25.81$ [2.23]) and control group 1 (postmean $=23.07$ [3.32]), and a strong effect (effect size $=1.19$; $95 \%$ confidence interval, 0.43 to 1.94 ) between the goalsetting group and control group 2 (postmean $=22.0$ [3.83]).

- Furthermore, we calculated Hedge $g$ effect sizes to determine the magnitude of the difference between posttreatment and pretreatment self-efficacy scores within each group included in Coppack et al. ${ }^{8}$ Our results indicated a strong positive effect size for the goal-setting group (effect size $=1.27$; $95 \%$ confidence interval, 0.51 to 2.03 ), a weak positive effect size for control group 1 (effect size $=0.08 ; 95 \%$ confidence interval, -0.61 to 0.77 ), and a weak positive effect size for control group 2 (effect size $=0.20 ; 95 \%$ confidence interval, -0.50 to 0.89 ).

\section{Results of Evidence Assessment}

The maximum score that each study could receive on the Physiotherapy Evidence Database scale was 10. A score $\geq 6$ indicates moderate- to high-quality evidence. ${ }^{11}$ Evans and Hardy ${ }^{7}$ received a score of 8 out of 10 , whereas Coppack et al $^{8}$ received a score of 9 out of 10. Evans and Hardy ${ }^{7}$ did not conceal allocation. Neither article blinded the therapists who were administering the intervention.

\section{Clinical Bottom Line}

There is currently consistent, good-quality, patient-oriented evidence that supports the use of goal setting-enhanced rehabilitation to improve self-efficacy in patients undergoing rehabilitation for sports-related injury compared with the standard of care group.

Strength of recommendation: The grade of $\mathrm{A}$ is recommended by the Strength of Recommendation Taxonomy for consistent, good-quality, patient-oriented evidence. ${ }^{12}$ 
Table 1 Characteristics of Included Studies

\begin{tabular}{ll}
\hline Study authors & $\begin{array}{l}\text { Coppack, Russell J. } \\
\text { Kristensen, Jakob } \\
\text { Karageorghis, Costas } \mathrm{I}^{8}\end{array}$ \\
& $\begin{array}{l}\text { Use of a goal setting intervention to increase adherence } \\
\text { to low back pain rehabilitation: a randomized controlled trial }\end{array}$ \\
& Randomized controlled trial \\
Study design & UK military personnel volunteers $(\mathrm{n}=48)$ with a diagnosis \\
Study & of nonspecific low back pain between the ages of 18 and \\
participants & 48 y were divided into 3 groups: \\
& Experimental group: goal setting and exercise therapy \\
& C1: therapist-led exercise therapy \\
& C2: non-therapist-led exercise therapy \\
& A total of 48 participants participated: age $=32.9(7.9) \mathrm{y}$
\end{tabular}

Inclusion/ exclusion criteria

Intervention investigated

Outcome measures Results

Level of

evidence

Physiotherapy

Evidence

Database score

Support for

the answer

Standardized 3-wk (5 d/wk, 15-d intervention) rehabilitation in accordance with physical ability.

Experimental group goal-setting intervention consisted of encouragement from supervising therapist for motivation and encouragement, and supervising therapist only monitored exercise technique to ensure safety.

SIRAS, SIRBS, and Biering-Sørensen test (Behavioral Regulation in Exercise Questionnaire)

The posttreatment score for self-efficacy in the experimental ysis showed a significant group $\times$ time effect for self-efficacy, $F_{2,44}=10.66\left(P<.001, \eta_{\mathrm{p}}^{2}=.33\right)$. Self-efficacy (SIRBS) was significantly higher in experimental group (25.81 [2.23], $P<.05)$ compared with both $\mathrm{C} 1$ (23.07 [3.23], $P<.05)$ and $\mathrm{C} 2(22.00[3.83], P<.05)$.

1B

$9 / 10$

Yes

\section{Evans, Lynne \\ Hardy, Lew ${ }^{7}$}

Injury rehabilitation: a goal setting intervention study

Randomized controlled trial

Participants $(n=39)$ were individuals who sustained a sports injury that required an operative procedure between the ages of 17 and $39 \mathrm{y}$ and were divided into 3 groups:

GS group: goal setting with sport psychologist and rehabilitation program

SSC group: meetings with sport

psychologist and rehabilitation program

C group: rehabilitation program

A total of 39 participants participated: age $=25.42(5.32) \mathrm{y}$

Inclusion: sustained sports injury precluding their participation in normal training/competition for a minimum of $5 \mathrm{wk}$, attendance at one of 2 sports injury clinics, and injury severity requiring operative procedure

Exclusion: those who could not be matched across the 3 groups 5-wk rehabilitation program for all 3 groups based on nature program with goal-setting intervention. All 3 groups completed a program consisting of individual and group-based submaximal, incremental exercise. Exercises performed in each session varied negotiating goals with sports psychologist and ranking goals on a scale of $1-10$ based on importance. $\mathrm{C} 1$ subjects received verbal coaching to correct exercise technique. $\mathrm{C} 2$ did not receive verbal group exhibited negative skewness. Follow-up univariate anal-

$1 \mathrm{~B}$

Yes

Abbreviations: C, control; C1, control group 1; C2, control group 2; DMRC, Defense Medical Rehabilitation Centre; GS, Experimental; SIRAS, Sport Injury Rehabilitation Adherence Scale; SIRBS, Sports Injury Rehabilitation Beliefs Survey; SSC, Social support control.

\section{Implications for Practice, Education, and Future Research}

The results of this CAT revealed consistent, good-quality, patientoriented evidence that supports the use of goal setting-enhanced rehabilitation to improve self-efficacy following sports-related injury. Coppack et $\mathrm{al}^{8}$ conducted a randomized controlled trial exploring the use of a 3-week goal setting-enhanced rehabilitation intervention to increase adherence to low back pain rehabilitation. Forty-eight military personnel with a diagnosis of nonspecific, nonoperative low back pain were randomly assigned to either a goal setting-enhanced rehabilitation experimental group, a therapistled exercise therapy group, or a non-therapist-led exercise therapy group. At preintervention and postintervention, participants completed the SIRBS to assess self-efficacy and treatment efficacy and the Biering-Sørensen test to assess functional outcome. Participants 
in all 3 groups completed an exercise program consisting of individual and group-based exercises. The goal-setting experimental group generated several priority goals with the guidance of a sports psychologist, and participants ranked these goals according to their own perceived importance; this formed a personal goal profile for each participant. The profile was a baseline for each participant, and goals were added to the profile throughout the rehabilitation process. Two follow-up meetings between each participant and the sports psychologist were conducted. At these meetings, new goals were added and/or repeated based on the progress of the participant. The therapist-led exercise group completed their exercise program with verbal encouragement and were monitored for proper exercise technique by the supervising therapist. The non-therapist-led exercise group did not have verbal encouragement from a therapist to motivate the participants. The therapists only monitored technique to ensure the safety of the participants. Following intervention, the goal-setting group exhibited significantly increased levels of self-efficacy (25.81 [2.23]) compared with both the social-support control group (23.07 [3.23], $P<.05)$ and the control group $(22.00$ [3.83], $P<.05)$.

Evans and $\mathrm{Hardy}^{7}$ conducted a randomized controlled trial examining the effects of a 5-week goal-setting intervention on athletes' rehabilitation adherence, self-efficacy, treatment efficacy, and overall psychological response to injury. Thirty-nine participants were matched across groups according to attending physiotherapist, nature of the injury, rehabilitation stage, sport, level of participation, and gender. Participants were randomly assigned to either a goal-setting intervention group, a social support group, or a control group. Injury included knee ligamentous injuries, shoulder dislocations, and lower leg fractures, and all required an operative procedure. As in Coppack et al, ${ }^{8}$ self-efficacy was assessed using the SIRBS. The SIRBS was completed at 5-day intervals for 5 weeks. The participants in the goal-setting intervention group met with a sports psychologist every 7 to 10 days for 5 weeks to implement the goal-setting intervention. All goals were specific to the tasks associated with their musculoskeletal rehabilitation and were negotiated between the participant and the sports psychologist. The participants in the social support group met with a sports psychologist every 7 to 10 days for 5 weeks. The purpose of these sessions was to control for the social support interaction that occurred between the sports psychologist and the participants in the goal-setting group. The only contact the sports psychologist had with the control group was 5-minute phone calls every 10 days in order to encourage adherence to the study. The results of the Evans and Hardy ${ }^{7}$ study support the use of goal setting to enhance selfefficacy, because the goal-setting group demonstrated the greatest increase in self-efficacy at postassessment. Furthermore, selfefficacy increased over time for the goal-setting group, whereas both control groups showed a decrease in self-efficacy over time.

While there were strong effect sizes identified for both studies, the most important difference between the 2 interventions was the type of goals created throughout the rehabilitation process. Evans and $\mathrm{Hardy}^{7}$ utilized process and performance goals. Performance goals are goals that provide the building blocks needed to achieve an overall outcome goal (eg, perform a single-leg squat). ${ }^{13}$ Process goals are smaller goals that need to be achieved to attain performance goals (eg, range of motion). ${ }^{13}$ Successful completion of process and performance goals enhances the likelihood of achieving outcome goals (eg, return to sport). ${ }^{13}$ Coppack et $\mathrm{al}^{8}$ utilized goals that participants deemed fundamental and important for successful rehabilitation. Participants were assisted in generating goals and were then asked to rate them based on perceived importance. Rather than selecting goals directly associated with rehabilitation exercises, goals were created by the participants based on what they believed they needed to achieve in order to be successful at the end of their rehabilitation. The process by which Coppack et $\mathrm{al}^{8}$ implemented the goal-setting intervention encouraged participants to adhere to the program, because they were able to choose goals that they thought were important. It is suggested that yielding the control to the participant throughout the goal-setting process promotes self-efficacy, improving rehabilitation outcomes. ${ }^{8}$

These different types of goal-setting techniques provide implications for goal setting in athletic training clinical practice. First, athletic trainers should guide patients to develop appropriate goals. As demonstrated in both studies, it is important to work with patients to develop goals rather than independently developing goals for patients in order to appropriately enhance self-efficacy after sportsrelated injury. Second, it is important to ensure that the goals developed are important to the patient. Goal setting should be a negotiation between the patient and the clinician. ${ }^{7,8}$ If patients have a sense of autonomy with regard to their rehabilitation goals, then they will be more likely to adhere to their rehabilitation and experience an increase in self-efficacy. ${ }^{7,8}$ Last, it is important to utilize an array of goals during rehabilitation to enhance selfefficacy. An outcome goal of return to sport should be divided into performance and process goals, because achieving smaller goals will promote self-mastery in the patient, lead to more goal achievement, and ultimately lead to increased levels of self-efficacy. ${ }^{4}$

In addition to implementing goal-setting techniques, it is important that athletic trainers consider the use of patient-reported outcome measures to examine the effectiveness of their goalsetting interventions. ${ }^{7,8}$ The SIRBS $^{9}$ is a patient-reported outcome measure that was used across both studies to measure self-efficacy with acceptable internal consistency and validity. ${ }^{9}$ The SIRBS 9 uses a 7-point Likert scale ranging from "very strongly disagree" to "very strongly agree." This instrument contains a 4-item subscale that was specifically designed to assess self-efficacy. Higher scores represent higher levels of self-efficacy. Implementation of a 4-item subscale is time efficient and can provide clinicians with important information that can help guide their clinical practice. However, while the objective score is useful, it is also important to examine the instrument subjectively in order to make decisions for patient care. Clinicians should identify specific questions for which the patient may have reported low levels of self-efficacy in order to begin a conversation about the patient's confidence and provide insight into appropriate goals to develop with the patient in order to improve self-efficacy.

Finally, the results of these studies demonstrate the importance of interprofessional collaboration. Coppack et $\mathrm{al}^{8}$ and Evans and Hardy $^{7}$ included a sports psychologist in the implementation of the goal-setting interventions in their respective studies. It is important to appreciate the positive impact that interprofessional collaboration can have on improving health outcomes after sports-related injury. However, a sports psychologist may not always be readily available to collaborate with athletic trainers to implement goal-setting techniques. It has been demonstrated that rehabilitation specialists, including athletic trainers, can implement psychoeducation techniques like goal setting in order to improve health outcomes within their clinical practice. ${ }^{14}$ Therefore, it is important that athletic trainers utilize these competencies in implementing goal setting within their clinical practice in order to address deficits in self-efficacy. If left unaddressed, self-efficacy may lead to poor outcomes despite the integration of appropriate physical rehabilitation techniques. 
Future research should explore the optimal timing of goal setting to increase self-efficacy following sports-related injury. This research will provide insight into when and how often to adjust goals throughout the rehabilitation program in order to achieve optimal outcomes. In addition, future research should further examine the effects of goal setting on the rehabilitation of different types of injuries, ranging from acute to chronic, mild to severe, and operative versus nonoperative. The studies summarized in this CAT included patients with a variety of injuries, some of which warranted surgical intervention while other patients did not undergo surgery and completed rehabilitation exercises. The implementation of goal setting to effectively increase self-efficacy may differ between various sports-related injuries and treatment paradigms. In addition, future research should explore the impact of incorporating different types of goals (ie, process and performance goals) that are considered to be important to the patient. This should also include the number of goals and the time to achieve goals as covariates in the analyses.

Deficits in self-efficacy can negatively affect health outcomes after sports-related injury. Although this area has been less explored in the musculoskeletal population, cognitive behavioral intervention and psychoeducation can help to enhance health outcomes. The results of this CAT demonstrate that goal setting is an effective psychoeducation intervention for enhancing selfefficacy. This CAT should be reviewed in 2 years (2021) to determine whether additional evidence is present that can provide further insight into the use of goal setting to enhance self-efficacy following sports-related injury.

\section{References}

1. Burt CW, Overpeck MD. Emergency visits for sports-related injuries. Ann Emerg Med. 2001;37(3):301-308. PubMed ID: 11223767 doi:10. 1067/mem.2001.111707

2. Everhart JS, Best TM, Flanigan DC. Psychological predictors of anterior cruciate ligament reconstruction outcomes: a systematic review. Knee Surg Sports Traumatol Arthrosc. 2013;23(3):752762. PubMed ID: 24126701 doi:10.1007/s00167-013-2699-1

3. Thomee P, Wahrborg P, Borjesson M, Thomee R, Eriksson BI, Karlsson J. Self-efficacy, symptoms and physical activity in patients with an anterior cruciate ligament injury: a prospective study. Scand J Med Sci Sports. 2007;17(3):238-245. PubMed ID: 16774652
4. Bandura A. Self-efficacy: toward a unifying theory of behavioral change. Psychol Rev. 1977;84(2):191-215. PubMed ID: 847061 doi:10.1037/0033-295X.84.2.191

5. Woby SR, Urmston M, Watson PJ. Self-efficacy mediates the relation between pain-related fear and outcome in chronic low back pain patients. Eur J Pain. 2007;11(7):711-718. PubMed ID: 17218132 doi:10.1016/j.ejpain.2006.10.009

6. Dohnke B, Knäuper B, Müller-Fahrnow W. Perceived self-efficacy gained from, and health effects of, a rehabilitation program after hip joint replacement. Arthritis Care Res. 2005;53(4):585-592. doi:10. 1002/art.21324

7. Evans L, Hardy L. Injury rehabilitation: a goal-setting intervention study. Res $Q$ Exerc Sport. 2002;73(3):310-319. PubMed ID: 12230338 doi:10.1080/02701367.2002.10609025

8. Coppack RJ, Kristensen J, Karageorghis CI. Use of a goal setting intervention to increase adherence to low back pain rehabilitation: a randomized controlled trial. Clin Rehabil. 2012;26(11):1032-1042. PubMed ID: 22357799 doi:10.1177/0269215512436613

9. Taylor AH, May S. Threat and coping appraisal as determinants of compliance with sports injury rehabilitation: an application of Protection Motivation Theory. J Sports Sci. 1996;14(6):471-482. PubMed ID: 8981286 doi:10.1080/02640419608727734

10. Rosenthal R. Parametric measures of effect size. In: Cooper H, Hedges LV, eds. The Handbook of Research Synthesis (pp. 231244). New York, NY: Russell Sage Foundation.

11. Husted JA, Cook RJ, Farewell VT, Gladman DD. Methods for assessing responsiveness: a critical review and recommendations. $J$ Clin Epidemiol. 2000;53(5):459-468. PubMed ID: 10812317 doi:10. 1016/S0895-4356(99)00206-1

12. Ebell MH, Siwek J, Weiss BD, et al. Strength of recommendation taxonomy (SORT): a patient-centered approach to grading evidence in the medical literature. Am Fam Physician 2004;69(3):548-556. PubMed ID: 14971837

13. Covassin T, Beidler E, Ostrowski J, Wallace J. Psychosocial aspects of rehabilitation in sports. Clin Sports Med. 2015;34(2):199-212. PubMed ID: 25818709 doi:10.1016/j.csm.2014.12.004

14. Baez S, Hoch MC, Hoch JM. Evaluation of cognitive behavioral interventions and psychoeducation implemented by rehabilitation specialists to treat fear-avoidance beliefs in patients with low back pain: a systematic review. Arch Phys Med Rehabil. 2018; 99(11):2287-2298. PubMed ID: 29247627 doi:10.1016/j.apmr. 2017.11 .003 